\title{
Commentaire sur l'Exposé de Karl Borch
}

\author{
par Hugo Clemeur *
}

Le Professeur Karl Borch nous a démontré, dans son exposé, de manière claire et évidente l'existence de trois terrains très différents sur lesquels s'exerce cette activité que nous appelons l'assurance, c'est-à-dire, le terrain de l'assurance-vie, celui de l'assurance des particuliers et des ménages et enfin le terrain de l'assurance des entreprises.

L'implacable logique de cet exposé nous conduit à penser qu'il est peut-être urgent de revoir, à la lumière des idées exposées par le Professeur Borch, certains concepts et certaines attitudes traditionnelles qui se rencontrent parfois encore dans quelques compagnies d'assurance qui approchent les différents marchés de l'assurance sans discernement. Il faut toutefois convenir qu'à l'heure actuelle un nombre important d'entreprises ont déjà largement tiré les conséquences des idées exposées et traduisent dans leur structure, comme dans leur action et leur système de direction, le concept de trois marchés d'assurance différents.

Dans le courant de l'exposé du Professeur Borch, on a pu relever au passage que celui-ci considère la réassurance comme un cas limite de l'assurance des entreprises. Peut-être y a-t-il sur ce point quelques nuances à apporter.

Pour le praticien de l'assurance il est clair qu'il existe un marché de la réassurance très réel et très actif. Il suffit de songer au rôle des réassureurs professionnels, à la concurrence qu'ils se livrent entre eux et à l'activité des courtiers de réassurance. L'existence de nombreux problèmes commerciaux, techniques, financiers et administratifs qui sont propres aux entreprises pour lesquelles la réassurance est l'activité principale constitue la preuve d'une spécificité évidente. De même sur le plan de la recherche actuarielle et économique pure, les problèmes de la réassurance, tant active que passive, ont toujours tenu une place importante dans les études.

On peut en conclure, sans hésiter, que la réassurance est une activité qui s'exerce suivant des règles particulières, et dans des conditions de fonctionnement telles que le marché de la réassurance est à considérer comme un marché à part entière.

$\mathrm{La}$ réassurance dépend de manière très étroite de l'activité de l'assurance directe. Ce serait donc une erreur d'affirmer que le marché de la réassurance que l'on vient de définir serait un marché totalement indépendant des trois autres.

* Katholieke Universiteit Leuven (K.U.L.). 
Il n'empêche que les particularités de ce marché sont suffisamment distinctes des particularités des autres marchés, pour qu'il puisse sans aucun doute être considéré comme un quatrième marché qui a un rôle spécifique à remplir.

$\mathrm{Au}$ niveau de l'assurance directe, l'existence de trois marchés distincts comporte des conséquences qui sont parfois plus fondamentales qu'on ne le croit. En effet, et quel que soit l'angle sous lequel on examine les caractéristiques de chacun de ces marchés, on se rend aisément compte de différences essentielles dans les conditions de fonctionnement. Le Professeur Borch a cité très justement quelques-unes des facettes de l'activité d'assurance qui nécessitent une approche différente suivant les marchés. Il a évoqué en premier lieu les questions de la distribution et du marketing des produits d'assurance. Il va de soi que le développement d'une stratégie commerciale cohérente ne peut se concevoir sans tenir compte des caractéristiques de la demande qui sont propres pour chaque marché.

En réalité, il n'y a pas que les aspects commerciaux qui sont concernés par l'existence de marchés différents. Les multiples aspects de la gestion technique sont, en réalité, tout aussi concernés. Il suffit, à cet égard, de songer aux problèmes d'organisation interne des entreprises d'assurance, à la définition des structures, des méthodes de direction et des gestions des services, aux moyens de contrôle interne, aux objectifs à atteindre et ainsi de suite. Cette différenciation ne concerne pas que les aspects administratifs de la gestion technique mais tout autant l'aspect actuariel à proprement parler qui doit s'adapter aux différences entre les trois marchés. Il suffit de citer les problèmes que posent la tarification des risques, la sélection, ainsi que la détermination des facteurs explicatifs sur lesquels sont basées les différenciations dans les tarifs, sans oublier bien entendu toutes les questions qui concernent l'évaluation des réserves techniques, et les modalités financières de leur couverture.

Le Professeur Borch a également évoqué le rôle particulier des autorités de contrôle et la nécessité d'avoir une philosophie d'approche différente suivant les marchés d'assurance concernés. Sur ce plan nous ne pouvons que nous féliciter des efforts de compréhension qui sont déployés par les différentes autorités tant sur le plan national que sur le plan supra-national, afin de mieux saisir les problèmes pratiques auxquels sont confrontées les sociétés d'assurance dans des marchés d'ailleurs fort turbulents à l'heure actuelle. Mais peut-être n'est-on pas encore allé assez loin dans ce domaine.

Sur le plan de l'assurance-vie par exemple, les autorités de contrôle ont une tâche réelle à remplir dans le contrôle de l'exactitude technique et actuarielle des opérations et des transactions. Il nous semble toutefois qu'elles pourraient contribuer, davantage encore que maintenant, à rendre l'épargne au moyen de l'assurance-vie aussi concurrentielle que possible par rapport aux autres possibilités d'épargne et d'investissement qui sont offertes au public. En effet, le Professeur Borch a démontré de façon magistrale la supériorité des formules d'épargne qu'offre l'assurance-vie au point de vue de son utilité sociale et économique. Il serait par conséquent non seulement souhaitable mais donc aussi logique que les autorités de contrôle s'attachent à supprimer ou du moins à écarter les entraves de tout ordre qui freinent le développement harmonieux de la branche vie. A titre d'exemple, une simplification des formalités administratives entraî- 
nées par les exigences de diverses autorités permettrait sans aucun doute de revoir les chargements de gestion et d'administration nécessaires à l'heure actuelle et permettrait à l'assurance-vie de mieux se mesurer aux autres formules d'investissement et d'épargne offertes par des organismes de collecte de l'épargne, concurrentes des sociétés d'assurance-vie. Sur d'autres plans encore, tels que le régime des investissements, ou le régime fiscal, des efforts seraient souhaitables.

Sur le plan du marché de l'assurance des particuliers, des malaises subsistent encore qui s'expliquent en partie du moins par une information insuffisante du grand public sur le rôle et les modalités de l'assurance. Certaines pratiques de quelques compagnies d'assurance de seconde zone n'y sont peut-être pas entièrement étrangères d'ailleurs. La mission des autorités de contrôle sur ce terrain est claire. Il s'agit de promouvoir et de soutenir des formules d'assurance techniquement saines, présentant une protection efficace pour les particuliers et les ménages, et pour lesquels la concurrence entre les sociétés d'assurance ne s'effectue plus sur le seul niveau du prix mais bien sur le niveau de la qualité des services et de la couverture offerte.

Enfin, sur le plan des assurances des entreprises, il est évident, comme le fait remarquer le Professeur Borch, que les assureurs n'ont pas besoin d'aide pour se défendre dans ce marché. Si les assureurs n'ont pas besoin d'aide, il ne faut pas non plus que les autorités leur enlèvent les armes dont ils ont besoin pour défendre une position qui est parfois loin d'être confortable devant certains interlocuteurs de taille.

On peut relever entre autres que certaines entreprises d'assurance se trouvent parfois singulièrement démunies de moyens d'action pour démonter de vastes opérations de fraude à l'assurance. Dans cet ordre d'idées, il serait certainement intéressant de s'interroger, dans les modèles théoriques des marchés de l'assurance, sur le phénomène de la fraude à l'assurance et d'étudier quels en sont les mécanismes. Il n'est pas exclu que l'œuvre du Professeur Laffont ouvre une brèche fort intéressante et prometteuse dans ce domaine, resté traditionnellement hermétique à toute recherche scientifique.

Une troisième remarque concerne le rôle des courtiers dans le marché de l'assurance. Dans le cours de son exposé, le Professeur Borch nous affirme que la présence des courtiers d'assurance est susceptible de rendre le marché de l'assurance plus optimal encore au sens de Pareto. Qu'est-ce que la théorie nous apprend " de facto » sur ce point ? Apparemment fort peu de choses. L'immense majorité des modèles, en effet, se limite à étudier des problèmes d'optimalisation de la distribution des primes et des risques entre deux agents ou groupes d'agents économiques, c'est-à-dire d'une part les assurés et d'autre part les compagnies d'assurance.

La pratique nous apprend toutefois que les choses sont moins simples et que ce serait une erreur que de sous-estimer, voire négliger, le rôle que joue le courtier dans la conclusion des contrats d'assurance. Ce rôle est-il positif ou est-il négatif ? La réponse à cette question n'est pas évidente. De nouvelles recherches pourraient explorer ce terrain relativement vierge et où, selon toute vraisemblance, il faudra faire ceuvre d'imagination pour intégrer dans un seul et même modèle les motivations et les comportements de chacune des trois parties. Selon une toute autre approche, on pourrait 
également s'interroger sur la question de savoir s'il existe, au point de vue de l'optimalisation des marchés, une différence notable entre les pays où le rôle du courtier dans la distribution de l'assurance est prépondérant, et les pays où le courtier n'existe virtuellement pas. A l'intérieur de l'Europe, des exemples de chacun des deux types de pays peuvent certainement être trouvés.

En conclusion il faut admettre que nous sommes encore loin de pouvoir apporter la solution théorique à tous les problèmes de l'assurance. Il en va certainement de même sur d'autres terrains de l'activité économique. Grâce toutefois aux nombreuses études des pionniers mais également des chercheurs actuels, on peut affirmer aujourd'hui que l'assurance possède sans aucun doute ses lettres de noblesse fondées sur des bases scientifiques très réelles.

Cependant, il continue à subsister un écart certain entre la théorie de l'assurance et sa pratique. On peut se demander quelles en sont les raisons.

Contrairement à certaines sciences telles que la physique nucléaire où l'on a commencé par trouver les formules et où l'on a construit les centrales après, en assurance, on a commencé par faire de l'assurance et ce n'est qu'après qu'on a commencé à se poser des questions. Ce fait historique explique peut-être en partie le fait qu'il subsiste souvent encore dans la recherche théorique sur l'assurance une ambiguïté quant aux conclusions des études et des modèles. En effet, les modèles proposés par la recherche sont-ils à caractère normatif, c'est-à-dire des modèles qui doivent servir de base à une prise de décision ou, cherche-t-on, au contraire, à construire des modèles descriptifs, c'est-à-dire des modèles qui se limitent à dresser une épure des transactions et des comportements courants et normaux dans l'assurance. Il faut avouer qu'il existe parfois de la confusion entre les deux.

Ce n'est que depuis les années 60 que les milieux qui s'intéressent aux fondements scientifiques du phénomène de l'assurance, se sont enfin rendus compte de ce que le problème principal à résoudre consiste à pénétrer le comportement humain devant l'incertitude et d'en étudier les conséquences et les retombées dans la vie pratique des ménages et des entreprises, et dans celle des compagnies d'assurance. C'est le grand mérite du Professeur Borch d'avoir mis le doigt sur cette question cruciale et d'y avoir apporté des solutions originales et enrichissantes. 